\title{
Wavelength Self-Managed Optical WDM Source Using Polarization-Multiplexed Fabry-Pérot Laser Diodes
}

\author{
Jin-Serk Baik, Kun-Youl Park, Tae-Won Oh, and Chang-Hee Lee, Member, IEEE
}

\begin{abstract}
We propose and demonstrate a novel wavelength self-managed optical source for access networks. By polarization multiplexing with two Fabry-Pérot laser diodes, we can largely reduce wavelength and polarization dependence on the injected amplified spontaneous emission (ASE) light. The worst case power penalty incurred by the wavelength detuning can be decreased within $1 \mathrm{~dB}$. We can obtain not only increased signal power and signal-to-noise ratio but also widened operation range. Even though polarized ASE light is used, data transmission is possible using the proposed source. Therefore, the proposed source is very practical to use in low-cost optical wavelength-division-multiplexing access networks.
\end{abstract}

Index Terms-Fabry-Pérot (FP) lasers, optical access network, optical fiber communication, polarization multiplexing, wavelength-division multiplexing (WDM).

\section{INTRODUCTION}

$\mathbf{T}$ HERE HAVE been many trials to implement a cost-effective wavelength-division-multiplexing (WDM) source for optical access networks [1]-[4]. Among them, the wavelength-locked Fabry-Pérot laser diode (FP LD) with external narrow-band amplified spontaneous emission (ASE) injection is attractive because of its wavelength self-management characteristics [1]. The injected ASE light forces FP LD to operate as a quasi-single-mode oscillation and suppresses its mode partition noise. However, the transmission performance depends on the wavelength detuning between the injected sliced ASE and a lasing mode of the LD. As we gradually change the temperature of the LD, the bit-error-rate (BER) performance fluctuates periodically. This can be solved by using the LD packaged with a thermoelectric cooler; however, the system cost is inevitably increased in this case. We can also use wide-band ASE light injection with a flat-top arrayed-waveguide grating (AWG) [2]. A reflective semiconductor optical amplifier can be used as a solution for wavelength independence operation [3]. However, this device is not yet cost-efficient for optical access networks.

Moreover, we need an unpolarized ASE light to have polarization-independent operation, since FP LD has inherent polarization-dependent internal gain. Therefore, we cannot use a polarized ASE, e.g., a high-power super-luminescent diode (SLD), as an injection light. If we want to employ the SLD, a polarization-independent source will be required.

Manuscript received May 7, 2004; revised June 4, 2004.

The authors are with the Department of Electrical Engineering, Korea Advanced Institute of Science and Technology, Daejeon 305-701, Korea (e-mail: leo@photonet.kaist.ac.kr).

Digital Object Identifier 10.1109/LPT.2004.833905

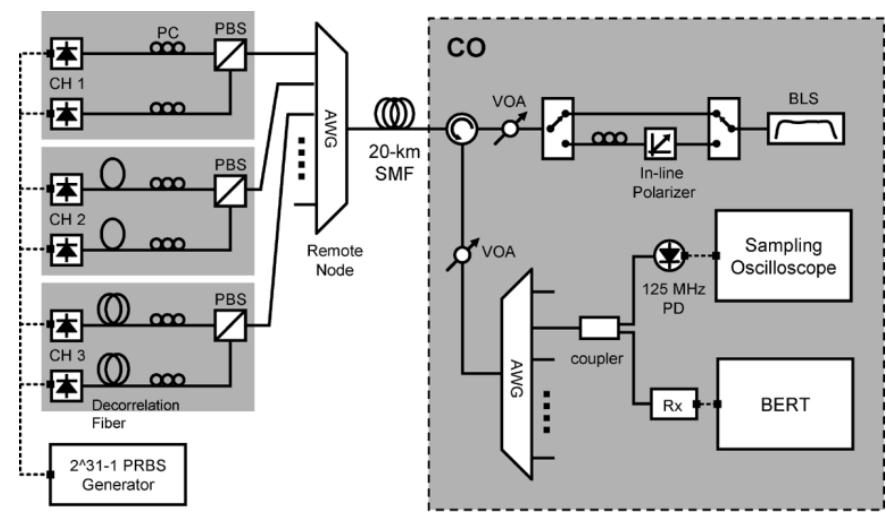

Fig. 1. Experimental setup for three-channel transmission with the proposed polarization-multiplexed sources. (VOA: variable optical attenuator. BLS: broad-band light source. BERT: BER tester).

In this letter, we propose and demonstrate a new novel configuration of polarization-multiplexed FP LDs to eliminate both the temperature and the polarization dependence from the previous wavelength-locked FP LDs. We show that the worst-case power penalty can be reduced within about $1 \mathrm{~dB}$, even if the locking effect is degraded by temperature variation. The proposed source also shows good immunity from the polarization state of injected polarized light. In addition, we can extend the operational wavelength range of the proposed source by using FP LDs of different gain peak wavelengths. Therefore, the proposed source is very practical to use in low-cost optical access networks.

\section{EXPERIMENTAL RESULTS}

The experimental setup is shown in Fig. 1. The proposed source is implemented by using a pigtailed polarization beam splitter (PBS), polarization controllers (PCs), and two FP LDs. We used three pairs of FP LDs for WDM transmission. All LDs have $600-\mu \mathrm{m}$ cavity length and gain peak at $1545 \pm 1 \mathrm{~nm}$. They are antireflection coated with $1 \% \sim 2 \%$ reflectivity on the front facet in order to increase the ASE injection efficiency and reduce the reflected power. An erbium-doped fiber-based ASE is used for incoherent injection light at the central office (CO). This ASE is transmitted through 20-km single-mode fiber (SMF), filtered by nonflattop AWG located at the remote node, split by the PBS according to polarization, and injected into each LD that located at the subscribers side. The channel spacing and the 3-dB bandwidth of the AWG are $100 \mathrm{GHz}$ and $0.4 \mathrm{~nm}$, respectively. The wavelengths of WDM signals are 1552.4, 1553.2, 


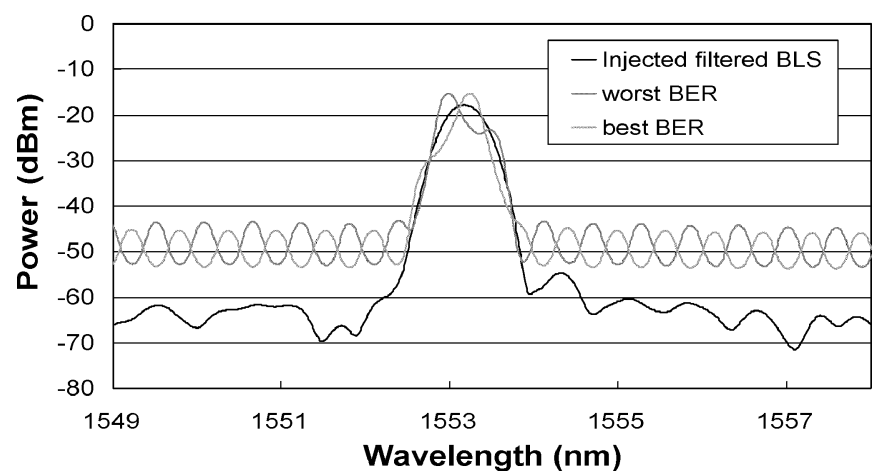

Fig. 2. Injected AWG-filtered BLS and wavelength-locked FP LD output spectra in which the best BER and worst BER can be obtained, respectively.
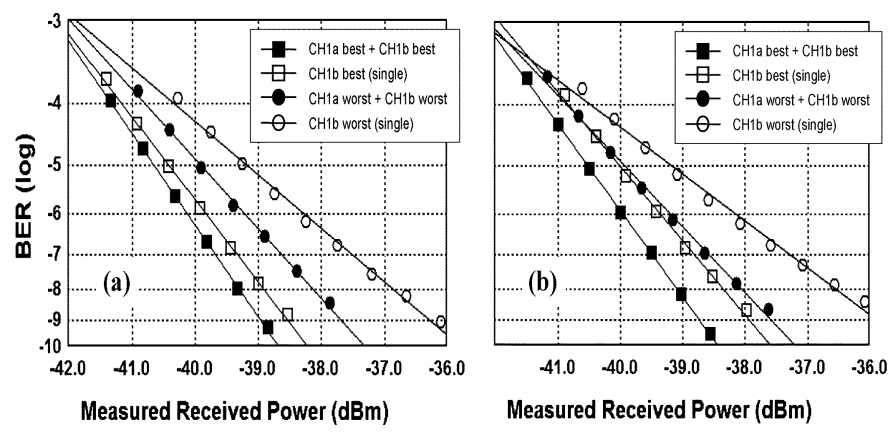

Fig. 3. (a) Measured BER curves with polarization multiplexed FP LDs when $-18-\mathrm{dBm} / 0.2-\mathrm{nm}$ BLS power is injected. (b) Measured BER curves with the proposed source when $-22-\mathrm{dBm} / 0.2-\mathrm{nm}$ BLS power is injected.

and $1554.0 \mathrm{~nm}$. The injected polarized ASE power at the input of the each FP LD was $-18 \mathrm{dBm} / 0.2 \mathrm{~nm}$ at peak. The output polarization of each LD was aligned to the polarization axis of the corresponding PBS port by using PC. We modulate the LDs with $155-\mathrm{Mb} / \mathrm{s}$ pseudorandom data, and transmit them together along 20-km SMF to the CO. The BER of each channel was measured at the CO.

First of all, we examine temperature-dependent properties of the proposed source as a function of the difference between the wavelengths of a LD mode and the injected ASE. In Fig. 2, we can see that the wavelength-locked laser output has different spectra according to the LD temperature. By sweeping the wavelength of the LD mode by tuning its temperature, we can find the specific wavelengths occurring the best (lowest) BER and the worst (highest) BER under a fixed LD bias and a constant injected ASE power. In the case of a single wavelength-locked FP $\mathrm{LD}$, the sensitivity difference between those two BER curves was more than $2.3 \mathrm{~dB}$ at $10^{-9} \mathrm{BER}$, as shown by the hallowmarked curves in Fig. 3(a). The solid-marked curves in Fig. 3(a) represent the performance of the proposed polarization-multiplexed source. The BER curves of the best and the worst cases are improved because the effective signal-to-noise ratio (SNR) is increased by $3 \mathrm{~dB}$ at the same power resulted from the two-LD multiplexing. The power penalty between both curves is reduced by $1.2 \mathrm{~dB}$ even though both LDs are in the worst BER condition. When either LD is in the best BER condition while the other is in the worst, the penalty becomes almost negligible. Typically, the lasing positions of two FP LDs are likely to be different from each other at the same temperature. Therefore, we can neglect

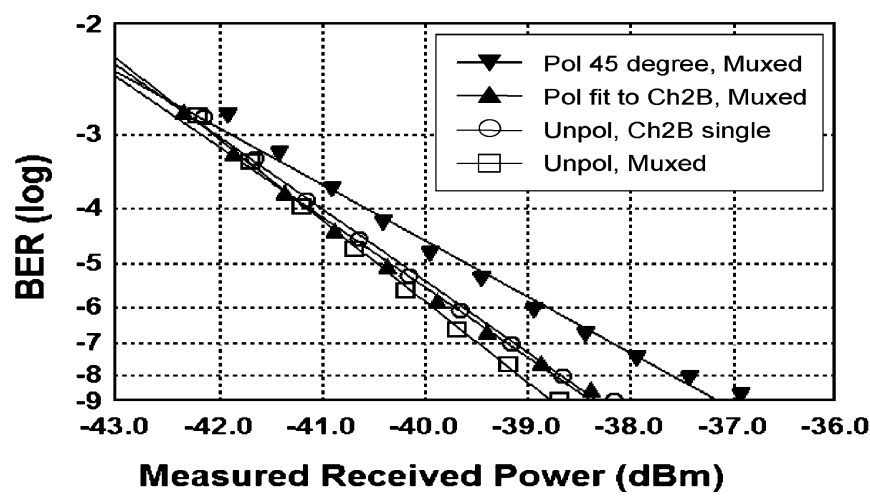

Fig. 4. Comparison between the cases of unpolarized ASE injection (hallow markers) and polarized ASE injection (solid markers).

the penalty incurred by the temperature variation with the proposed source.

We also examine the effect of injection power variation. At $-22-\mathrm{dBm} / 0.2-\mathrm{nm}$ injection power [Fig. 3(b)], we got $0.5-\mathrm{dB}$ power penalty at the best case compared with Fig. 3(a). The BER is degraded drastically even at the best case if we inject ASE power of less than $-22 \mathrm{dBm} / 0.2 \mathrm{~nm}$. However, by using the proposed source, we can obtain an error-free transmission. We can also see that the penalty between the best and the worst curves remains within $1 \mathrm{~dB}$ at $-22-\mathrm{dBm} / 0.2-\mathrm{nm}$ injection power.

In order to examine the polarization dependence, we tested both the unpolarized and the polarized ASE as the injection light. In the case of a single FP LD, the output has strong dependence on the polarization of injected ASE. If we inject orthogonally polarized ASE (transverse-magnetic mode of the laser) into the single FP LD, we cannot see any injection effects at all. Thus, this is not able to be used as a WDM source. However, with the proposed source which operates as an unpolarized laser, we can eliminate the polarization dependence, as shown in Fig. 4. Since we fixed the injected ASE power, the total ASE power after the inline polarizer is reduced by half. The polarization axis of the polarized ASE is aligned to the TE polarization axis of either one of the LDs or inclined by $45^{\circ}$ with respect to that axis of each LD. The proposed source in the latter case shows $1.5-\mathrm{dB}$ power penalty at $10^{-9}$ BER relative to the case with unpolarized ASE, because the power of injected ASE into each LD becomes half of the unpolarized ASE. Even though that case makes some penalty, we demonstrated error-free transmission. In other words, we reduce dramatically the polarization dependence rather than the case using only a single LD.

We also measured the influence of crosstalk by adjacent channels in WDM transmission. As shown in Fig. 5, there is no penalty compared with the single-channel transmission. Thus, the crosstalk from the WDM transmission is negligible.

The wavelength-locking efficiency is getting degraded as the wavelength of injected ASE is going away from the gain envelope peak wavelength of an LD. In the case of a single LD, we have 1-dB degradation of sidemode suppression ratio (SMSR) on the operation range of $\pm 6 \mathrm{~nm}$ from the peak. To widen the operation range of the proposed WDM source, we used two LDs whose gain peak wavelengths are different from each other. The gain peak wavelengths were 1530 and $1555 \mathrm{~nm}$, respectively. 


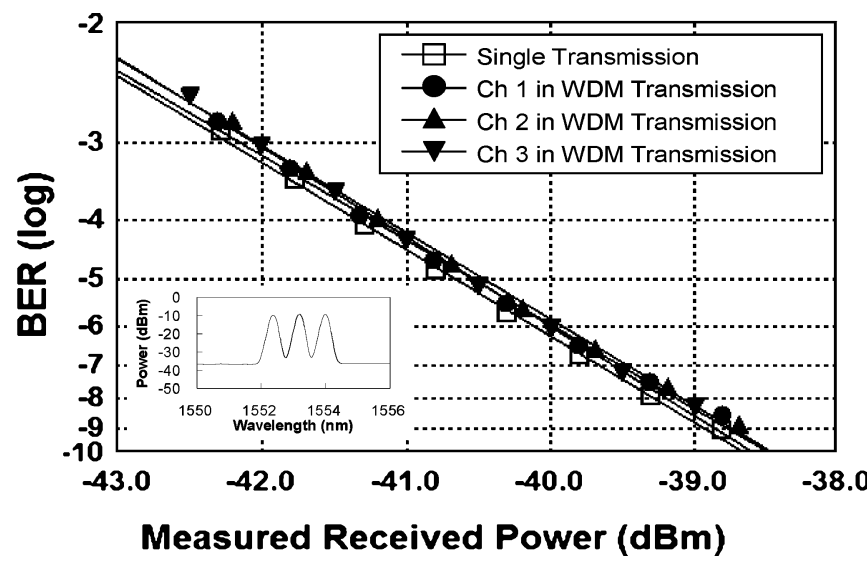

Fig. 5. WDM transmission curves compared with a single LD transmission.

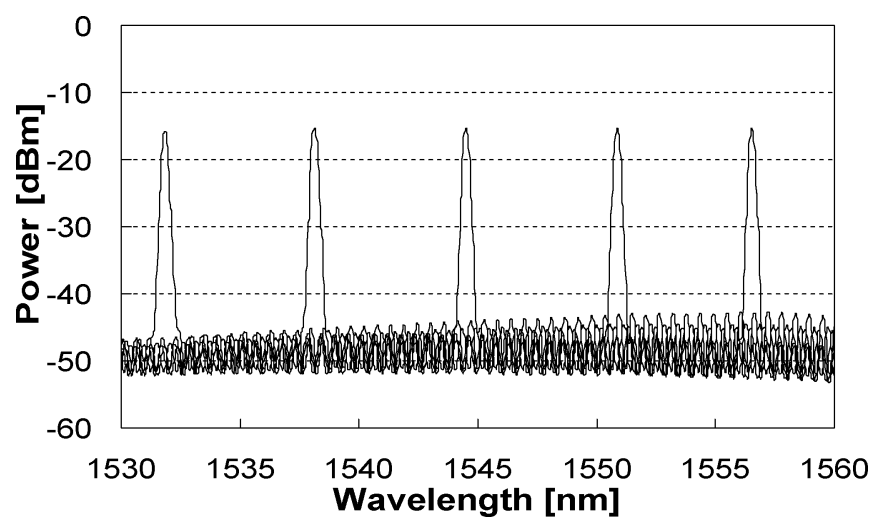

Fig. 6. Tuning property of the proposed source with two lasers that have different envelope peak wavelengths.

As shown in Fig. 6, the proposed source shows almost a constant output power over 25-nm wavelength range from 1531.9 to $1556.5 \mathrm{~nm}$. The SMSR stays more than $29 \mathrm{~dB}$ which is sufficiently large to use as a WDM source. This result is valid only with the unpolarized ASE injection. If we use polarized ASE and the LDs with different gain peaks, the tuning range will be reduced compared with a single LD.

\section{DISCUSSION AND CONCLUSION}

The cost of the proposed source is likely to be increased, since we should use two LDs and a polarization multiplexer. However, it may be minimized by implementing this source in a small packaging that includes a spatial walkoff prism or a PBS as the polarization multiplexer. Additionally, if we add a pair of WDM filters and PDs, then we can make a new polarization-independent bidirectional (BiDi) module. We illustrate this schematic in Fig. 7. It may be noted that we can eliminated a polarization-dependent characteristics of the filter, since only polarized light passes through each filter. This feature helps to reduce the

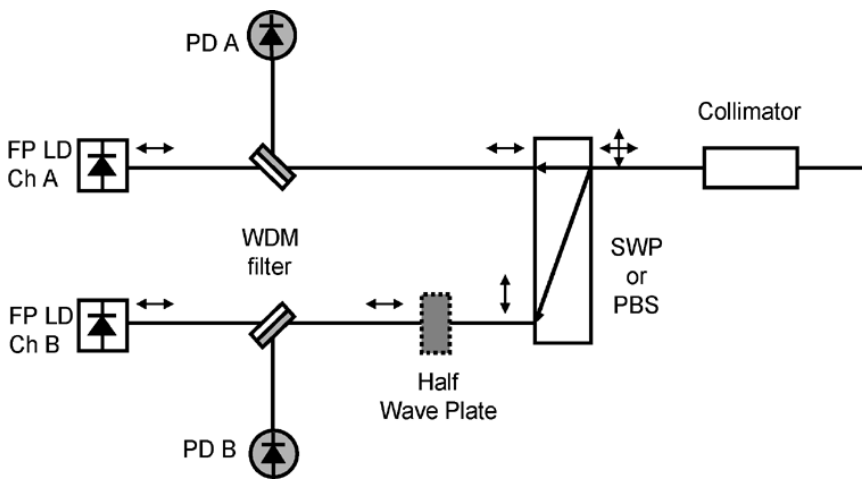

Fig. 7. BiDi module consists of the proposed source and a pair of WDM filters and PDs implemented in a single package.

guard band of the filters between two transmission bands, i.e., the upstream and the downstream.

The proposed BiDi module can provide a few more advantages with unpolarized ASE injection. The two PDs can be configured as a balanced receiver to enhance the sensitivity when we use this module for both the upstream and the downstream. Moreover, we can increase reliability of the system, since either LD operates as a backup of the other one. Also, we utilize both polarization of the injected light to increase the SNR and the operation range.

In conclusion, we have proposed and demonstrated a novel WDM optical source using polarization-multiplexed two FP LDs with external ASE injection. We can use polarized injection light with the proposed source. This source has the properties of increased SNR, doubled output power, and raised ASE power utilization for unpolarized injected light. It is also tolerant against the wavelength misalignment due to temperature variation. We also show it has negligible penalties in WDM transmission and possibly has wide tuning range by using the LDs of different gain peak. Therefore, the proposed source is suitable for a low-cost and high-performance WDM source in cost effective optical access networks.

\section{REFERENCES}

[1] H. D. Kim, S.-G. Kang, and C.-H. Lee, "A low-cost WDM source with an ASE injected Fabry-Pérot semiconductor laser," IEEE Photon. Technol. Lett., vol. 12, pp. 1067-1069, Aug. 2000.

[2] D. J. Shin, D. K. Jung, J. K. Lee, J. H. Lee, Y. H. Choi, Y. C. Bang, H. S. Shin, J. Lee, S. T. Hwang, and Y. J. Oh, " 155 Mbit/s transmission using ASE-injected Fabry-Pérot laser diode in WDM-PON over $70^{\circ} \mathrm{C}$ temperature range," Electron. Lett., vol. 39, no. 18, pp. 1331-1332, Sept. 2003.

[3] P. Healey, P. Townsend, C. Ford, L. Johnston, P. Townley, I. Lealman, L. Rivers, S. Perrin, and R. Moore, "Spectrum slicing WDM-PON using wavelength-seeded reflective SOAs," Electron. Lett., vol. 37, no. 19, pp. 1181-1182, Sept. 2001.

[4] D. K. Jung, S. K. Shin, C.-H. Lee, and Y. C. Chung, "Wavelength-division-multiplexed passive optical network based on spectrum-slicing techniques," IEEE Photon. Technol. Lett., vol. 10, pp. 1334-1336, Sept. 1998.

[5] H.-L. Althaus, W. Gramann, W. Reill, and W. Spath, "Optoelectronic module for bidirectional optical data transmission," U.S. Patent US6 097521 , Aug. 2000.

[6] M. Ogusu, T. Tomioka, and S. Ohshima, "Receptable-type optical transmission/reception apparatus," U.S. Patent US6 040 934, Mar. 2000. 